\title{
EL PATRIMONIO COMO ELEMENTO DINAMIZADOR DE LA SOCIOECONOMÍA DE LAS COMUNIDADES LOCALES EN EL MARCO DE LAS POLIITICAS EUROPEAS. PROPUESTA DE ACCIONES Y ACTUACIONES TURÍSTICAS
}

\author{
M.J. Viñals Blasco \\ I. Martínez-Sanchis \\ P. Alonso-Monasterio \\ Universitat Politècnica de València \\ mvinals@upv.es, immarsan.arq@gmail.com, ambpau@gmail.com
}

\section{RESUMEN}

El patrimonio natural y cultural puede constituir un pilar para la dinamización socioeconómica de ciertos territorios, especialmente de la mano del sector turístico. Este trabajo se centra en el análisis de los Fondos Estructurales y de Inversión Europeos (EIE), concretamente en el análisis del objetivo temático 6: Conservar y proteger el medio ambiente y promover la eficiencia de los recursos, Prioridad de Inversión c: la conservación, la protección, el fomento y el desarrollo del patrimonio natural y cultural del Fondo de Desarrollo Regional (FEDER). Así, se reflexiona en torno a las directrices y requerimientos de este objetivo para orientar las líneas de acción en base a la dinamización turística del patrimonio.

Palabras clave: Patrimonio, Turismo, Desarrollo Local, Fondos Estructurales y de Inversión Europeos.

\section{ABSTRACT}

The natural and cultural heritage can be a pillar for the socioeconomic revitalization of certain territories, leaning especially in the tourism sector. This paper focuses on the analysis

Fecha de recepción: julio 2015.

Fecha de aceptación: marzo 2016. 
of the European Structural and Investment Funds, especially on the basis of their Thematic Objective number 6: Preserving and Protecting the Environment and Promoting Resource Efficiency, Investment Priority c: Conserving, Protecting, Promoting and Developing Natural and Cultural Heritage, of the European Regional Development Fund. Hereby, it addresses the guidelines and requirements for this objective to provide a basis for heritage tourism opportunities.

Keywords: Heritage, Tourism, Local Development, EU Structural and Investment Funds.

\section{INTRODUCCIÓN}

Para abordar los objetivos de este trabajo se hace necesaria una introducción en relación a los instrumentos de financiación de la Unión Europea ya que son los que pautan las estrategias y las temáticas sobre las prioridades de inversión. Por ello, para encajar acciones y actividades relacionadas con el binomio patrimonio-turismo procede conocer dónde quedan enmarcadas y cuáles son los principios que rigen el desarrollo de las mismas para la programación europea en curso (2014-2020).

Los Fondos Estructurales y de Inversión de la Unión Europea (Fondos EIE) tienen como objetivo promover la competitividad y la convergencia de todos los territorios y son la principal fuente de inversión de la UE para ayudar a los Estados miembros a restablecer y aumentar el crecimiento y garantizar una recuperación que genere empleo, al tiempo que garantizan el desarrollo sostenible, en consonancia con los objetivos y aplicación de la estrategia Europa 2020 (Comisión Europea, 2010).

La Estrategia Europa 2020 constituye un marco estratégico para la acción de las administraciones públicas nacionales y regionales (objetivos, directrices, acciones, etc.) para el decenio 2010-2020. Las bases de esta estrategia son la cohesión económica, social y territorial. Propone tres prioridades: 1) el crecimiento inteligente (desarrollo de una economía basada en el conocimiento y la innovación), 2) el crecimiento sostenible (promover una economía que utilice más eficazmente los recursos, más verde y competitiva) y 3 ) el crecimiento integrador (fomento de una economía con alto nivel de empleo que tenga cohesión económica, social y territorial).

Esta Estrategia incluye 11 objetivos estratégicos que se reflejan en el artículo 9 del Reglamento UE No $1303 / 2013$ de las Disposiciones Comunes de los Fondos EIE y que son:

1) Potenciar la investigación, el desarrollo tecnológico y la innovación;

2) Mejorar el uso y la calidad de las tecnologías de la información y de las comunicaciones y el acceso a las mismas;

3) Mejorar la competitividad de las pequeñas y medianas empresas, del sector agrícola y el sector de la pesca y la acuicultura;

4) Favorecer la transición a una economía baja en carbono en todos los sectores;

5) Promover la adaptación al cambio climático y la preservación y gestión de riesgos;

6) Conservar y proteger el medio ambiente y promover la eficiencia de los recursos; 
7) Promover el transporte sostenible y eliminar los estrangulamientos en las infraestructuras de red fundamentales;

8) Promover la sostenibilidad y la calidad en el empleo y favorecer la movilidad laboral;

9) Promover la inclusión social y luchar contra la pobreza y cualquier forma de discriminación;

10) Intervenir en educación, formación y formación profesional para la adquisición de capacidades y un aprendizaje permanente;

11) Mejorar la capacidad institucional de las autoridades públicas y las partes interesadas y la eficiencia de la administración pública.

El conjunto de los Fondos Estructurales y de Inversión (EIE) lo constituyen: el Fondo Europeo de Desarrollo Regional (FEDER), el Fondo Social Europeo (FSE), el Fondo de Cohesión, el Fondo Europeo Agrícola de Desarrollo Rural (FEADER) y el Fondo Europeo Marítimo y de Pesca (FEAMP). El Tratado de Funcionamiento de la Unión Europea asigna objetivos claros a estos instrumentos, y señala la necesidad de estar coordinados entre ellos. Durante el periodo de programación 2014-2020, se estableció un nuevo marco legislativo para estos fondos. Así, por una parte están las Disposiciones Comunes transversales a todos ellos y, además, un reglamento específico para cada fondo ${ }^{1}$.

En el Reglamento UE N¹303/2013 de Disposiciones Comunes para los EIE, se prevé la adopción de Contratos de Asociación que establezcan los compromisos de los socios a nivel nacional y regional, creando un Marco Estratégico Común que fija las metas estratégicas y unas prioridades de inversión claras (programas) para cada Estado para el período de programación 2014-2020, permitiendo así una mejor sinergia entre todos los fondos para maximizar su impacto (fig. 1).

El contexto en el que se encuadra este trabajo es el Acuerdo de Asociación de España 2014-2020 (Ministerio de hacienda y Administraciones Públicas, 2014) que es un documento elaborado a partir de un proceso conjunto de participación y coordinación entre el Estado Español y las regiones, en el que se definen, analizan y desarrollan 10 de los 11 objetivos temáticos definidos en el Reglamento UE No1303/2013 relativos a la Estrategia 2020 antes mencionada (el undécimo no se ha tratado de forma sectorial en este documento). En particular interesa a los efectos de este trabajo, el objetivo temático 6 (OT6): Conservar y proteger el medio ambiente y promover la eficiencia de los recursos. En el documento se encuentra un diagnóstico previo respecto a los diferentes objetivos que sirven como base para justificar las propuestas de intervención. En lo que respecta al objetivo 6, se presenta por tanto, un análisis de la importante riqueza patrimonial y biodiversidad española y la necesidad de conservar las áreas naturales protegidas, en particular las pertenecientes a la Red Natura 2000.

1 Fondo Europeo de Desarrollo Regional (Reglamento UE n ${ }^{\circ}$ 1301/2013)

Fondo Social Europeo (Reglamento UE n⿳ 1304/2013)

Fondo de Cohesión (Reglamento UE nº 1300/2013)

Fondo Europeo Agrícola de Desarrollo Rural (Reglamento UE n ${ }^{\circ} 1305 / 2013$ )

Fondo Europeo Marítimo y de Pesca (FEAMP) (Reglamento UE nº 508/2014) 
Figura 1

ESQUEMA DE LA ESTRUCTURA QUE ARTICULA LOS FONDOS EUROPEOS DE LA ESTRATEGIA EUROPA 2020

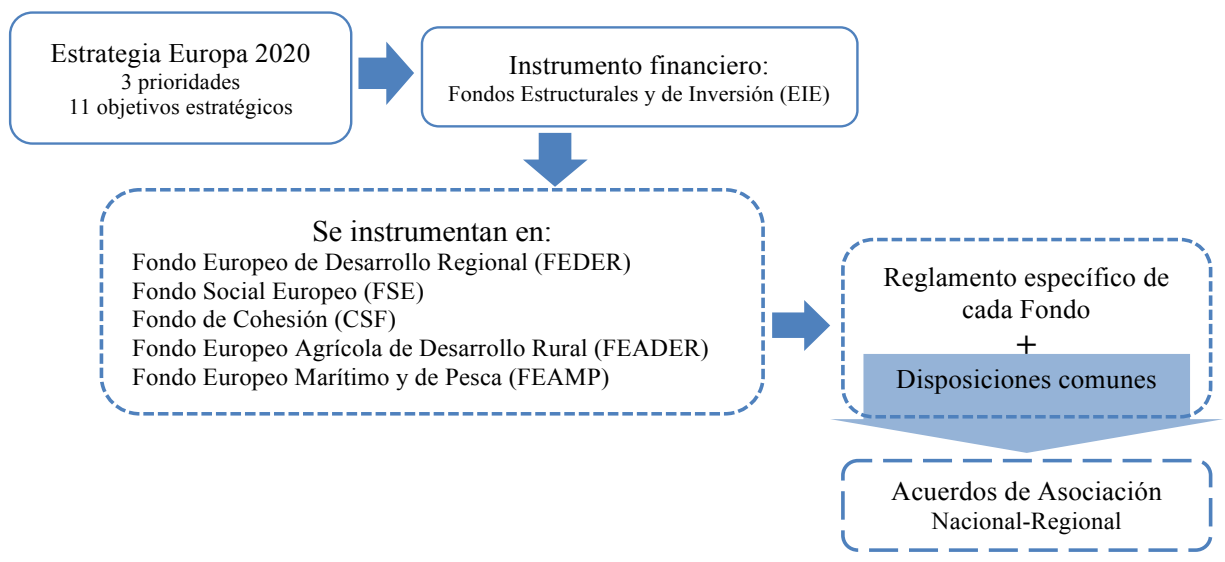

En relación a este objetivo, se identificaron básicamente una serie de propuestas de intervención referidas al patrimonio natural y cultural y la biodiversidad. De este modo, respecto al patrimonio se habla de invertir en infraestructuras orientadas a la protección y mejora del patrimonio para la ordenación del uso público y para el impulso de procesos de desarrollo local basados en la puesta en valor de dicho patrimonio, haciéndose especial referencia a la red de caminos rurales. Por lo que respecta a la biodiversidad, se considera prioritaria su protección y conservación en aplicación de las restantes políticas ambientales y, particularmente, se dedica especial atención a la Red Natura 2000.

En este documento de Acuerdo de Asociación de España 2014-2020, el objetivo temático OT6, antes mencionado, es asignado indicativamente a nivel nacional al Fondo Europeo de Desarrollo Regional (FEDER) que, como recordamos, su finalidad es fortalecer la cohesión económica y social en la Unión Europea, para corregir los desequilibrios entre sus regiones.

En paralelo al Acuerdo de Asociación, las Comunidades Autónomas han elaborado los Programas Operativos donde se recogen las líneas estratégicas a desarrollar y propuestas de inversión sobre la base de un diagnóstico de la situación socioeconómica regional.

El Reglamento UE Nº1301/2013 del Parlamento Europeo y del Consejo de 17 de diciembre de 2013 sobre el Fondo Europeo de Desarrollo Regional (FEDER) desarrolla las disposiciones específicas relativas al tipo de actividades que pueden financiarse con cargo de este fondo con el fin de contribuir a las prioridades de inversión correspondientes a los objetivos temáticos fijados en el Reglamento (UE) n 1303/2013 de Disposiciones Comunes. Así, en relación con el objetivo temático 6 (OT6): Conservar y proteger el medio ambiente y promover la eficiencia de los recursos, se proponen siete prioridades de inversión. Entre las que se vinculan al patrimonio (fig. 2), destaca especialmente: la $c$ que se focaliza en $l a$ conservación, la protección, el fomento y el desarrollo del patrimonio natural y cultural y, secundariamente, la $d$ que se dedica a la protección y el restablecimiento de la biodiversidad y del suelo y el fomento de los servicios de los ecosistemas, inclusive a través de Natura 2000 $y$ de infraestructuras ecológicas. 


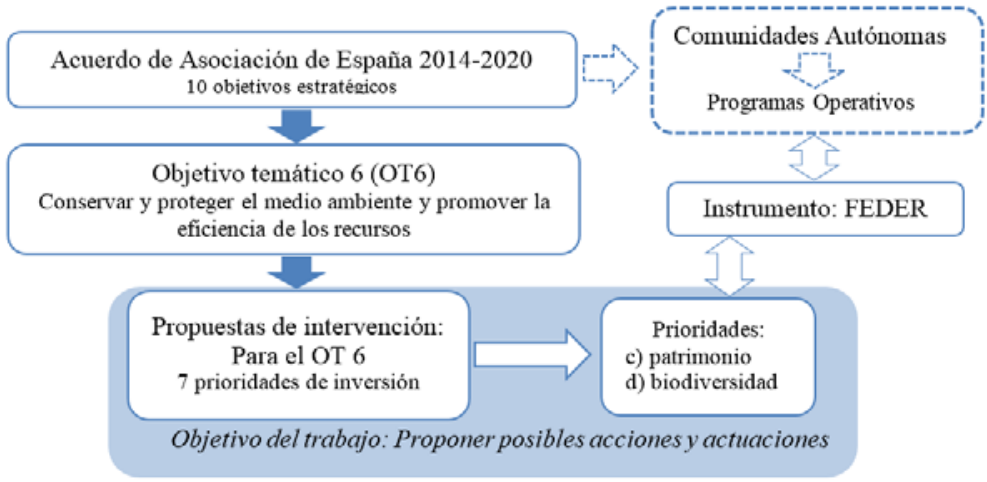

Este trabajo tiene como objetivo proponer posibles acciones y actuaciones en relación a este objetivo temático (OT6) relacionado con la conservación, protección, fomento y desarrollo del patrimonio natural y cultural europeo. Especial énfasis se dedica a la consideración del patrimonio como activo económico, especialmente turístico. Así, se observa que, desde su concepción tradicional inicial como elementos de la naturaleza o resultado de la creación humana al que hay que proteger y encapsular para su conservación, se ha pasado por la consideración de recurso que satisfaga una necesidad humana (hedonista, cultural, etc.), o por aquella que focaliza la atención en el valor de legado patrimonial. Todas estas percepciones coexisten actualmente, y se observan cada vez con más interés, como se ha comentado, con la visión del patrimonio como motor económico. Existen numerosos estudios de las últimas dos décadas sobre los resultados y el impacto económico del turismo cultural que avalan el éxito de estas propuestas, entre ellos destacan aquellos llevados a cabo por instituciones como la Organización para la Cooperación y el Desarrollo Económicos (OECD, 2009), el Consejo Internacional de Monumentos y Sitios Histórico-Artísticos (ICOMOS, 2011), el Programa de Naciones Unidas para el Medio Ambiente y la Organización Mundial del Turismo (UNEP y UNTWO, 2012), o la propia Unión Internacional para la Conservación de la Naturaleza (Osipova et al., 2014).

\section{METODOLOGÍA}

El método de trabajo que se ha seguido para el desarrollo de este trabajo ha consistido en una revisión en profundidad de todos los documentos estratégicos y reglamentos de los Fondos Estructurales y de Inversión de la Unión Europea para el periodo 2014-2020 que ya han sido enunciados en el apartado anterior. También se ha llevado a cabo un análisis del documento de Acuerdo de Asociación de España (2014-2020) y de los Planes Operativos de las diversas Comunidades Autónomas que lo han hecho público.

Desde el punto de vista del patrimonio natural y cultural, se parte de la experiencia académica y profesional en el campo de la conservación y gestión del patrimonio de las autoras y se 
hace un análisis y selección de las diferentes acciones y actuaciones posibles, presentándolas de forma ordenada, estructurada y secuencial para dotarlas de la necesaria coherencia a la hora de su posible programación y ejecución. De este modo, se presenta un primer bloque de acciones y actuaciones en relación a la conservación y protección que deben ser las primeras en ser implementadas, para posteriormente desarrollar las relativas a la puesta en valor y, finalmente, aquellas que tienen que ver con el fomento y dinamización socioeconómica del patrimonio.

Se ha prestado especial interés al estudio de los indicadores por ser un punto clave en la preparación de propuestas, ya que la Comisión Europea exige la identificación de herramientas evaluativas y de seguimiento claras para medir el grado del cumplimiento de los programas (indicadores de productividad) y también los resultados de los mismos (objetivos específicos).

Estas propuestas, tanto de acción y estrategia como de evaluación, son perfectamente extrapolables y podrían integrarse en los diversos planes integrales y de desarrollo local de cualquier territorio europeo que tengan el objetivo de generar empleo y desarrollar una economía basada en la conservación, protección y desarrollo sobre la base del patrimonio natural y cultural. Se intenta pues ofrecer una propuesta pragmática sobre el desarrollo de proyectos y programas que supere el discurso teórico y, definitivamente, focalice su atención sobre el potencial de los recursos patrimoniales, que por su carácter único e irrepetible acrecientan la competitividad territorial.

\section{ANTECEDENTES: LA DINAMIZACIÓN SOCIOECONÓMICA DEL PATRIMONIO}

El patrimonio natural, cultural y paisajístico son las señas de identidad de numerosas comunidades locales y la base para la dinamización de la socioeconomía de ciertos territorios. Esta dinamización ha ido, habitualmente, de la mano del sector turístico que, con mayor o menor acierto, ha catalizado el desarrollo de algunos lugares (García et al., 2016).

Con el paso del tiempo, esta situación necesita revisarse porque hace falta una renovación de las propuestas tradicionales que sea más acorde con las nuevas tendencias de la demanda (turismo interpretativo, turismo experiencial, etc.), porque pueden desarrollarse nuevos destinos que nunca antes habían formado parte de la oferta turística, y porque las actuales líneas de financiación de la Unión Europea apuntan básicamente a proyectos donde el patrimonio sea la base de la dinamización socioeconómica. Así pues, se considera que las diversas propuestas que encajen en la definición de "turismo sostenible" de la Organización Mundial de Turismo ${ }^{2}$ pueden ser consideradas seriamente como opciones viables.

La consideración del patrimonio como elemento dinamizador y articulador del territorio y como motor del desarrollo económico no es un asunto nuevo y numerosas organizaciones como la Unión Europea han debatido sobre este tema (Lähdesmäki, 2014). Desde la perspectiva del patrimonio natural, se observa que el Plan Estratégico del Convenio de Diversidad

2 El turismo sostenible es considerado aquel que tiene en cuenta las repercusiones actuales y futuras, económicas, sociales y medioambientales para satisfacer las necesidades de los visitantes, de la industria, del entorno y de las comunidades anfitrionas, dando un uso óptimo a los recursos medioambientales, manteniendo los procesos ecológicos esenciales y ayudando a conservar los recursos naturales y la diversidad biológica, respetando la autenticidad sociocultural de las comunidades anfitrionas, conservando sus activos culturales y arquitectónicos y sus valores tradicionales y asegurando unas actividades económicas viables a largo plazo reportando beneficios socioeconómicos. http://sdt.unwto.org/es/content/definicion 
Biológica 2011-2020 y los objetivos de Aichi y la Estrategia de la Unión Europea en materia de Biodiversidad (2011) apuntan a que el patrimonio natural y la biodiversidad son factores importantes de bienestar, de impulso socioeconómico y de articulación territorial. En lo que respecta al patrimonio cultural, hay que destacar la Declaración de París sobre el Patrimonio como motor de Desarrollo, adoptada en la 17 Asamblea General de ICOMOS en París (Francia, 2011). Por otra parte, está la inclusión, por primera vez, de la cultura en los Objetivos de Desarrollo Sostenible de la Agenda para el Desarrollo Sostenible 2030 durante la 70a Asamblea General de la ONU (Naciones Unidas, 2015). En el marco de la Unión Europea, destaca la Comunicación de la Comisión sobre la necesidad de una visión integrada del patrimonio cultural COM (2014) 477 (Comisión Europea, 2014). En este documento se ponen de relieve las diferentes herramientas transversales de la UE a nivel nacional y regional para apoyar la protección, puesta en valor intrínseco y social del patrimonio en aras de facilitar el crecimiento económico y la creación de empleo.

Lo que resulta más novedoso es que el patrimonio se contemple como objetivo de los EIE, en particular en los FEDER. De hecho, en los Planes Operativos aparece desarrollado entre los objetivos específicos adscritos a la prioridad de inversión 6c: 6.3.1. Promover la protección, fomento y desarrollo del patrimonio cultural, 6.3.2. Protección, desarrollo y promoción de las áreas naturales, en particular las de interés turístico y también el 6.3.4. Promover la protección, fomento y desarrollo del patrimonio cultural y natural de las áreas urbanas, en particular las de interés turístico. Llama la atención en esta prioridad de inversión, la inclusión del objetivo específico 6.3.3. Fomento de infraestructuras verdes que contribuyan al transporte alternativo en todo tipo de territorios y a la diversificación hacia actividades de bajas emisiones que tiene un carácter de movilidad no motorizada y también resulta llamativo la no consideración de los espacios protegidos naturales como activos turísticos ya que quedan relegados como parte (tanto desde el punto de vista temático como en relación al tipo de acciones exclusivamente de protección y gestión) del objetivo específico 6.4.1. Fomentar la gestión, protección y mantenimiento del suelo, de espacios naturales y su biodiversidad, en particular los protegidos, incluyendo medidas para paliar los problemas de erosión, salinización, desertificación, deforestación y bajo nivel de materia orgánica en el suelo, de la prioridad de inversión 6d: La protección y el restablecimiento de la biodiversidad y del suelo y el fomento de los servicios de los ecosistemas, inclusive a través de Natura 2000 y de infraestructuras ecológicas.

A partir del análisis de los campos de intervención mencionados para los FEDER (Ministerio de Hacienda y Administraciones Públicas, 2015), se identifican aquellos que podrían estar vinculados a esta prioridad de inversión 6c: en el área R06 Medio Ambiente están: CI089/CE091 Desarrollo y promoción del potencial turístico de los espacios naturales ${ }^{3}$, CI090/CE092 Protección, desarrollo y promoción de los activos del turismo público, CI091/CE093 Desarrollo y promoción de los servicios de turismo público, CI092/CE094 Protección, desarrollo y promoción de los activos de la cultura y el patrimonio públicos y CI093/CE095 Desarrollo y promoción de los servicios de la cultura y el patrimonio públicos. También podrían considerarse algunas áreas de R06 Naturaleza y Biodiversidad y R13 Desarrollo urbano.

3 Códigos de referencia: CI Campo de Intervención; CE Indicador común de productividad definido en la normativa comunitaria 
En este contexto, hay que destacar las potencialidades que presenta España para solicitar estos fondos ya que, por ejemplo, el valor de la biodiversidad española es el más elevado de la Unión Europea. Así, tenemos que según datos del Ministerio de Agricultura, Alimentación y Medio Ambiente ${ }^{4}$, la Red Natura 2000 en España está formada actualmente por 1.467 Lugares de Importancia Comunitaria (LIC) y por 644 Zonas de Especial Protección para las Aves (ZEPA), que comprenden en conjunto una superficie total de más de $210.000 \mathrm{~km}^{2}$ (27\% del territorio español). Para poder evaluar el potencial del patrimonio cultural español, sirvan de ejemplo los datos del Ministerio de Educación, Cultura y Deporte (2015) que cifraban el conjunto de inmuebles inscritos en el año 2014 como Bienes de Interés Cultural (BIC) en 17.107. Por su parte, el total de sitios inscritos en el Convenio de Patrimonio Mundial de la UNESCO asciende a $45^{5}$, con lo que España se posiciona en el tercer país del mundo con más bienes declarados. Además, está la red de Reservas de la Biosfera del Programa sobre el Hombre y la Biosfera (UNESCO) que, en 2016, estaba integrada por 48 sitios $^{6}$. No resulta ajeno pues pensar en el vínculo que existe entre esta relevante oferta patrimonial y el fenómeno turístico.

En vista de que se trata de un tema relevante a nivel nacional, en 2014, se presentó el Real Decreto 416/2014, de 6 de junio, por el que se aprobaba el Plan sectorial de Turismo de Naturaleza y Biodiversidad 2014-20207. Por otra parte, desde 2010 se han redactado distintos planes para canalizar el fenómeno del turismo cultural en nuestro país. Tal es el caso del Plan de Promoción Internacional del Turismo Cultural 2010-2012 ${ }^{8}$ (acuerdo interministerial de los Ministros de Cultura; Industria, Turismo y Comercio, y de Asuntos Exteriores y de Cooperación en 2009), y también el Plan Estratégico General 2012-2015 de la Secretaría de Estado de Cultura del Ministerio de Educación, Cultura y Deporte, (2012). De cualquier manera, hay que señalar que no hay resultados oficiales de la aplicación de todos estos planes.

\section{RESULTADOS: PROPUESTAS DE ACCIONES PARA INCLUIR EN LA PRIORIDAD DE INVER- SIÓN UE 6C: CONSERVACIÓN, PROTECCIÓN, FOMENTO Y DESARROLLO DEL PATRIMO- NIO NATURAL Y CULTURAL}

A la vista del marco normativo europeo, de las potencialidades del patrimonio español y de la conveniencia de emprender un nuevo modelo de acción territorial sostenible que sirva para impulsar nuevas iniciativas en relación al turismo de naturaleza y turismo cultural y para mejorar las ya existentes, el planteamiento de partida desde el que arranca este trabajo es analizar detalladamente el concepto expresado en la prioridad de inversión 6c de los Fondos FEDER considerando su potencial como herramienta de financiación. Así vemos que los verbos de acción utilizados en este enunciado son: conservar, proteger, fomentar y desarrollar.

4 http://www.magrama.gob.es/es/biodiversidad/temas/espacios-protegidos/red-natura-2000/rn_espana.aspx

5 http://whc.unesco.org/fr/list/stat\#d2

6 https://es.wikipedia.org/wiki/Anexo:Red_Mundial_de_Reservas_de_la_Biosfera

7 Real Decreto 416/2014, de 6 de junio, por el que se aprueba el Plan sectorial de turismo de naturaleza y biodiversidad 2014-2020. Ministerio de la Presidencia. «BOE» núm. 147, de 18 de junio de 2014. Referencia: BOEA-2014-6432

8 http://www.minetur.gob.es/es-es/gabineteprensa/notasprensa/documents/planpromocionturismointern.pdf 
La interpretación que se puede hacer de estos verbos de acción puede ser individualizada e independiente, o conjunta, interdependiente y secuenciada. Es decir, ¿Podrían en esta prioridad de inversión incluirse acciones y actuaciones dedicadas únicamente a la conservación? ¿Podrían incluirse acciones con una finalidad exclusivamente de protección legal, por ejemplo? ¿Se podrían proponer actuaciones solo de promoción? ¿En qué acciones de dinamización estamos pensando si el bien patrimonial no está protegido legalmente? La respuesta a estas preguntas no es evidente en los documentos normativos ni en los tutoriales o notas aclaratorias que emanan de la Comisión Europea. Solamente, la propuesta de la Comisión Europea de utilizar como indicador de productividad el C009 Aumento del número de visitas previstas a lugares pertenecientes al patrimonio cultural y natural y atracciones subvencionados (visitas/año) para este OT6 en los Programas Operativos (Ministerio de Hacienda y Administraciones Públicas, 2015), nos induce a pensar que se trata de llevar a cabo todo un conjunto de acciones encadenadas y secuenciales porque es la única forma en que pueden ciertamente desembocar en un resultado medible por este indicador de productividad.

La propuesta que se desarrolla en este trabajo va dirigida a identificar acciones y actuaciones que encajen en esta prioridad de inversión, pero no se trata solamente de proporcionar una amalgama o cluster de iniciativas, sino que es muy importante la secuencia con la que han de ser ejecutadas para que realmente se alcancen los resultados finales esperados.

De esta forma, se propone a continuación una secuencia de acciones organizada en torno a tres bloques temáticos que incluyen, a su vez, actuaciones, siguiendo el esquema de un plan operativo. Así, tenemos:

- Bloque 1: Conservación y protección del patrimonio natural y cultural

- Bloque 2: Puesta en valor turística del patrimonio natural y cultural

- Bloque 3: Dinamización socioeconómica turística del patrimonio natural y cultural

Las características específicas que presentan las acciones y actuaciones analizadas se deben a la propia naturaleza dinámica que caracteriza el territorio y al enfoque sistémico con el que debe abordarse este análisis para garantizar la necesaria transversalidad y considerar las complejas interdependencias que se producen entre naturaleza, patrimonio y turismo. De este modo, estas acciones deben estar sometidas a un continuo proceso de revisión con arreglo a los resultados obtenidos, modificándose si es necesario para obtener el impacto deseado. Por otra parte, debe existir una afinada coordinación entre las diversas administraciones ya que existe gran diversidad de elementos naturales y culturales a considerar en un mismo escenario territorial cuya responsabilidad gestora recae muchas veces en diferentes administraciones públicas. Esta acción de coordinación interadministrativa debe preceder a las relaciones que se establecerán con el sector privado.

Para garantizar un buen resultado final y, a pesar de que las acciones de cada bloque revisten un interés propio, no se podrán desarrollar acciones ni actuaciones del bloque 2 hasta que las del bloque 1 no estén suficientemente cumplimentadas y aplicadas, ni abordar la fase de dinamización socioeconómica (bloque 3) hasta que no se haya garantizado la conservación y protección de los recursos patrimoniales (bloque 1) y las actuaciones de accesibilidad física e intelectual del bloque 2. De este modo, todas las acciones y actuaciones deben estar contempladas desde una perspectiva integral, de conjunto y secuencial, sin olvidar la necesaria participación de las comunidades locales en la toma de decisiones. 


\section{IV.1. Acciones y actuaciones del bloque 1: Conservar / Proteger}

Las acciones que se proponen en el bloque 1 tienen que ver con la Preservación, Restauración, Rehabilitación, Conservación y Gestión activa de los recursos patrimoniales. Se considera que estas acciones son ineludiblemente las primeras a implementar para garantizar el buen estado de conservación del patrimonio. Están vinculadas a la prioridad de inversión 6c (conservación y protección del patrimonio).

En la tabla 1 se presentan acciones y actuaciones que van desde la protección legal de los elementos del patrimonio natural y cultural hasta las de sensibilización de la población local para abordar la dimensión social de la conservación del patrimonio, que es la más garantista a medio y largo plazo. Incluso se contempla la posibilidad de no utilizar un recurso (preservación) si tras una valoración intrínseca se considerara que la utilización de un bien patrimonial conllevaría un riesgo importante de degradación y/o pérdida del mismo.

Tabla 1

ACCIONES YACTUACIONES REFERIDAS A LA CONSERVACIÓN Y PROTECCIÓN DEL PATRIMONIO

\begin{tabular}{|c|c|}
\hline ACCIONES & ACTUACIONES \\
\hline \multirow{2}{*}{$\begin{array}{l}\text { 1.1. Garantizar la protección legal de } \\
\text { los elementos del patrimonio }\end{array}$} & 1.1.1. Creación de una figura de protección adecuada si no existe \\
\hline & 1.1.2. Aplicación de una figura de protección adecuada al sitio \\
\hline \multirow{4}{*}{$\begin{array}{l}\text { 1.2. Llevar a cabo una conservación } \\
\text { activa del patrimonio }\end{array}$} & $\begin{array}{l}\text { 1.2.1. Inventario y valoración intrínseca de los elementos del } \\
\text { patrimonio }\end{array}$ \\
\hline & $\begin{array}{l}\text { 1.2.2. Desarrollo de estándares de conservación de los elementos del } \\
\text { patrimonio }\end{array}$ \\
\hline & $\begin{array}{l}\text { 1.2.3. Restauración y/o rehabilitación de aquellos elementos del } \\
\text { patrimonio que lo precisen }\end{array}$ \\
\hline & $\begin{array}{l}\text { 1.2.4. Implementación de la protección física necesaria en aquellos } \\
\text { lugares donde sea necesario (vallados, etc.) }\end{array}$ \\
\hline $\begin{array}{l}\text { 1.3. Desarrollar herramientas técnicas } \\
\text { para la gestión de la conservación del } \\
\text { patrimonio }\end{array}$ & $\begin{array}{l}\text { 1.3.1. Redacción de un Plan de Gestión para la conservación } \\
\text { atendiendo a las indicaciones que se especifiquen en la figura de } \\
\text { protección aplicada }\end{array}$ \\
\hline \multirow{2}{*}{$\begin{array}{l}\text { 1.4. Prevenir, mitigar y corregir los } \\
\text { impactos que afectan al patrimonio }\end{array}$} & $\begin{array}{l}\text { 1.4.1. Adopción de medidas preventivas que eviten o amortigüen los } \\
\text { impactos }\end{array}$ \\
\hline & $\begin{array}{l}\text { 1.4.2. Impulso al desarrollo de actividades tradicionales que mantengan } \\
\text { activo el patrimonio y así, se mantenga en buenas condiciones }\end{array}$ \\
\hline \multirow{3}{*}{$\begin{array}{l}\text { 1.5. Incrementar la sensibilización } \\
\text { de la población residente y de } \\
\text { los visitantes (protección social) } \\
\text { para conseguir actitudes y } \\
\text { comportamientos proactivos de } \\
\text { aprecio por el patrimonio }\end{array}$} & $\begin{array}{l}\text { 1.5.1. Realización de campañas de sensibilización a las comunidades } \\
\text { locales sobre el valor del patrimonio }\end{array}$ \\
\hline & 1.5.2. Realización de actividades de sensibilización de los visitantes \\
\hline & $\begin{array}{l}\text { 1.5.3. Realización de códigos de buenas prácticas para el sector } \\
\text { turístico }\end{array}$ \\
\hline
\end{tabular}


Hay que destacar que este tipo de acciones en sí mismas no acaban de estar bien contempladas individualmente en los campos de intervención identificados en los FEDER, ya que las existentes siempre van ligadas a otras de promoción y desarrollo (CI090/CE092 Protección, desarrollo y promoción de los activos del turismo público y CI092/CE094 Protección, desarrollo y promoción de los activos de la cultura y el patrimonio públicos) y además los recursos patrimoniales presentan una consideración directa de activo turístico, pero estas acciones son imprescindibles para garantizar la conservación del patrimonio. También hay que señalar que, entre los campos de intervención, no aparece un área específica dedicada al patrimonio cultural, sino que van todas genéricamente incluidas en al área de "Medio Ambiente".

Por otra parte, hay que mencionar que el indicador de productividad C009 Aumento del número de visitas previstas a lugares pertenecientes al patrimonio cultural y natural y atracciones subvencionados (visitas/año), no sería posible aplicarlo en estos casos ya que los resultados de las acciones propuestas en este bloque no se pueden evaluar con el mismo. Todo ello, lleva a pensar, como ya se había avanzado, que este tipo de acciones, si bien absolutamente necesarias como base de cualquier iniciativa, no se pueden proponer si no van vinculadas a una secuencia concatenada de otras posteriores de promoción y desarrollo. No obstante, el indicador de objetivo específico E035 Número de personas impactadas por las actuaciones de difusión y sensibilización ambiental y de protección y desarrollo del patrimonio natural y cultural (Personas) sí podría utilizarse en relación a las acciones de sensibilización. Incluso se podría tomar como referencia el indicador específico E058 Superficie urbana afectada por operaciones de revitalización de las ciudades y de mejora del entorno urbano, su medio ambiente, o su patrimonio natural o cultural; incluidas en Proyectos pertenecientes a Estrategias Urbanas Integradas (Hectáreas), correspondiente al área de aplicación Desarrollo urbano (R13).

Otra cuestión interesante es que el patrimonio natural, en particular los espacios protegidos, no quedan incluidos en esta prioridad de inversión 6c, sino en la 6d de una manera más desdibujada y contemplando solo acciones de protección y gestión. En este caso concreto sí resultaría pertinente la utilización del indicador de productividad C023 Superficie de hábitats subvencionados para alcanzar un mejor estado de conservación (Hectáreas).

\section{IV.2. Acciones y actuaciones del bloque 2: Poner en valor}

Las acciones que se proponen en el bloque 2 sobre puesta en valor (tabla 2) no quedan mencionadas ni desarrolladas como tal en los FEDER, pero son un eslabón intermedio imprescindible entre las de conservación y las de dinamización socioeconómica del patrimonio.

La primera actuación propuesta consiste en inventariar, valorar e identificar los elementos del patrimonio que tienen un potencial de uso turístico. Posteriormente, se desarrollarán acciones y actuaciones para proporcionar acceso físico, intelectual y emocional al patrimonio. El acceso físico tiene que ver con el desarrollo de las necesarias adaptaciones para poder transitar y visitar el lugar en un marco de respeto a la integridad de los edificios (Heritage Office of NSW Department of Planning \& the Royal Australian Institute of Architects NSW, 2011; Catling, 2013) o del propio medio natural, y en condiciones de seguridad y confort físico y psicológico (Viñals et al., 2014). El acceso intelectual y emocional es fundamental para transmitir conocimiento sobre el lugar y para despertar en los visitantes sentimientos de aprecio (actitudes y comportamientos) por el patrimonio. 
Hay que mencionar que del tratamiento que se observa en los FEDER respecto al patrimonio, se interpreta directamente que va a ser utilizado para fines turísticos, especialmente visitas (a tenor del indicador de productividad propuesto anteriormente mencionado). A este respecto, hay que pensar que la mayoría de los bienes culturales no se diseñaron originalmente para ser objeto de visitas turísticas sino que detentaban una funcionalidad que en muchos casos ya no existe más. Por tanto, se trata, a menudo, de reutilizar edificios para otro fin diferente y por ello necesitan ser readaptados al nuevo uso turístico. Hay mucha literatura científica referida a este tema de la reutilización del patrimonio donde se propone la aplicación de unos principios de intervención respetuosos con la integridad y valores del edificio. También hay numerosos ejemplos exitosos de reutilización de edificios patrimoniales para usos turísticos que demuestran que los edificios se mantienen mejor cuando están en uso (Canada's Historic Places, 2010; Chapple, 2012).

Tabla 2

ACCIONES Y ACTUACIONES REFERIDAS A LA PUESTA EN VALOR DEL PATRIMONIO

\begin{tabular}{|c|c|}
\hline ACCIONES & ACTUACIONES \\
\hline \multirow{3}{*}{$\begin{array}{l}\text { 2.1. Desarrollar e } \\
\text { implementar herramientas } \\
\text { técnicas de gestión del uso } \\
\text { público }\end{array}$} & 2.1.1. Inventario y valoración recreativa de los elementos patrimoniales \\
\hline & $\begin{array}{l}\text { 2.1.2. Zonificación de los espacios, determinación de la capacidad de carga } \\
\text { recreativa y diseño del patrón de la visita }\end{array}$ \\
\hline & 2.1.3. Redacción, aprobación e implementación de un Plan de Uso Público \\
\hline \multirow{4}{*}{$\begin{array}{l}\text { 2.2. Facilitar el acceso } \\
\text { físico e intelectual y } \\
\text { emocional al patrimonio }\end{array}$} & $\begin{array}{l}\text { 2.2.1. Desarrollo y/o mejora de las infraestructuras y equipamientos básicos } \\
\text { mínimos para la visita (accesos rodados, aparcamientos, etc.) y aplicación de } \\
\text { criterios de diseño universal para todos en la medida de lo posible }\end{array}$ \\
\hline & 2.2.2. Redacción e implementación de Programas de Interpretación del Patrimonio \\
\hline & $\begin{array}{l}\text { 2.2.3. Desarrollo de equipamientos interpretativos (sistema señalización, senderos, } \\
\text { centros de interpretación, rutas interpretativas, museos, ecomuseos, centros de } \\
\text { información, etc.) }\end{array}$ \\
\hline & $\begin{array}{l}\text { 2.2.4. Formación a los recursos humanos dedicados al uso público (guías- } \\
\text { intérpretes, informadores, etc.) }\end{array}$ \\
\hline
\end{tabular}

El indicador de productividad común propuesto por los FEDER C009 Aumento del número de visitas previstas a lugares pertenecientes al patrimonio cultural y natural y atracciones subvencionados (visitas/año) podría considerarse válido para evaluar la eficiencia de la implementación de estas acciones. Algunos de los indicadores de objetivos específicos desarrollados en los FEDER podrían también aplicarse para la evaluación de estas acciones.

Especial atención se ha dedicado a la formación del personal de atención al público ya que se considera un elemento fundamental para establecer la conexión emocional que hay que crear entre el patrimonio y las personas y así convertir la actividad en una experiencia vivencial. 


\section{IV.3. Acciones y actuaciones del bloque 3: Promocionar / Desarrollar}

Las últimas acciones y actuaciones del bloque 3 (tabla 3) están vinculadas a la prioridad de inversión $6 \mathrm{c}$ que tienen que ver con el fomento y el desarrollo del patrimonio natural y cultural.

Tabla 3

\section{ACCIONES YACTUACIONES REFERIDAS A LA PUESTA EN VALOR DEL PATRIMONIO}

\begin{tabular}{|c|c|}
\hline ACCIONES & ACTUACIONES \\
\hline \multirow{3}{*}{$\begin{array}{l}\text { 3.1. Crear los } \\
\text { fundamentos para } \\
\text { desarrollar y dinamizar } \\
\text { un sistema turístico } \\
\text { basado en el patrimonio }\end{array}$} & $\begin{array}{l}\text { 3.1.1. Redacción e implementación de Planes Estratégicos para el desarrollo } \\
\text { turístico basados en el patrimonio natural y cultural }\end{array}$ \\
\hline & 3.1.2. Redacción e implementación de Planes de Dinamización Turística \\
\hline & $\begin{array}{l}\text { 3.1.3. Desarrollo de normativas legales para la regulación de actividades y } \\
\text { empresas turísticas vinculadas al patrimonio }\end{array}$ \\
\hline \multirow{4}{*}{$\begin{array}{l}\text { 3.2. Crear una oferta } \\
\text { turística de calidad } \\
\text { basada en el patrimonio }\end{array}$} & $\begin{array}{l}\text { 3.2.1. Identificación de los recursos patrimoniales con mayor potencial turístico } \\
\text { que constituyan la prioridad de inversión }\end{array}$ \\
\hline & $\begin{array}{l}\text { 3.2.2. Diseño y/o mejora de productos turísticos experienciales y diferenciados } \\
\text { basados en el patrimonio }\end{array}$ \\
\hline & $\begin{array}{l}\text { 3.2.3. Crear una cartera de productos turísticos a partir del ensamblaje de la oferta } \\
\text { de los diversos prestatarios turísticos }\end{array}$ \\
\hline & $\begin{array}{l}\text { 3.2.4. Apoyar el lanzamiento, desarrollo y/o la mejora de las empresas turísticas } \\
\text { (capacitación, incentivos fiscales, etc.) }\end{array}$ \\
\hline \multirow{2}{*}{$\begin{array}{l}\text { 3.3. Promocionar el } \\
\text { destino y los productos } \\
\text { turísticos patrimoniales }\end{array}$} & $\begin{array}{l}\text { 3.3.1. Identificar los rasgos esenciales de identidad y crear una imagen de marca } \\
\text { basada en los mismos }\end{array}$ \\
\hline & $\begin{array}{l}\text { 3.3.2. Redacción e implementación de un Plan de Comunicación Estratégica y de } \\
\text { Medios (online y offline) }\end{array}$ \\
\hline \multirow{3}{*}{$\begin{array}{l}\text { 3.4. Comercializar los } \\
\text { productos turísticos } \\
\text { patrimoniales }\end{array}$} & 3.4.1. Redacción e implementación de un Plan de Marketing \\
\hline & $\begin{array}{l}\text { 3.4.2. Implantación de nuevas tecnologías (TIC) de comercio electrónico } \\
\text { (e-commerce), sistemas de reserva, etc. }\end{array}$ \\
\hline & 3.4.3. Identificar la demanda potencial y diseñar estrategias para llegar a ella. \\
\hline
\end{tabular}

El primer aspecto que hay que mencionar es que, siguiendo los principios anteriormente expuestos de sistematicidad y secuencialidad, el orden en que se debe abordar la ejecución de las acciones y actividades debe considerar dar la prioridad en esta última fase a las de desarrollo y dejar para el final las de fomento. Se entiende en este trabajo que el fomento se asimila a acciones de promoción, divulgación y comunicación tal como se deriva de la versión inglesa del documento (promoting).

Por otra parte, hay que destacar que las acciones y actuaciones presentadas en las anteriores fases tienen un claro vínculo con las administraciones públicas ya que la gestión del patrimonio público, tanto natural como cultural, es una responsabilidad de las respectivas administraciones. En este tercer bloque, sin embargo, se incorpora el sector privado como claro protagonista ya que la dinamización y el desarrollo del sector turístico dependen fundamentalmente del empresariado, y en el caso de comunidades locales son sobre todo pequeñas 
y medianas empresas (PYMEs) y microPYMEs. El papel de la administración pública en estas acciones y actuaciones queda adscrito a la tutela y supervisión de la utilización que el sector haga de los elementos patrimoniales, así como de su promoción. Esto se lleva a cabo a través de planes (planes estratégicos y planes de dinamización), líneas directrices, reglamentos, etc. tal como se observa en las actuaciones vinculadas a la acción 3.1. "Crear los fundamentos para desarrollar y dinamizar un sistema turístico basado en el patrimonio" de este bloque (tabla 3 ).

El hecho de incorporar a las PYMEs en este contexto conlleva que algunas de estas acciones y actuaciones puedan recaer en la órbita del área de aplicación R01 Inversión productiva de los FEDER.

Las acciones de este bloque se consideran fundamentales para alcanzar el objetivo de considerar el patrimonio como un elemento dinamizador de la socioeconomía de las comunidades locales porque, a diferencia de las fases anteriores, en esta hay mayor probabilidad de creación de empleo estable y de generar réditos no dependientes directamente del asistencialismo de la administración pública.

Una de las acciones clave propuestas en este bloque es la 3.2. "Crear una oferta turística de calidad basada en el patrimonio". El sector turístico, que es a quién se apunta como motor de este proceso, se fundamenta en la venta de productos turísticos. Por ello, es necesario llevar a cabo un proceso mediante el cual, a partir de los recursos patrimoniales naturales y culturales, se defina una oferta turística viable y, de ahí, unos productos turísticos comercializables. Una actividad importante para orientar las prioridades de inversiones es identificar los recursos con mayor potencial turístico a través, por ejemplo, de un Índice de Potencialidad Turística (Viñals et al., 2016) para, de esta manera, orientar las inversiones y optimizar los recursos económicos disponibles.

Una vez se disponga de una cartera de productos turísticos, se procederá con las acciones de promoción en las que la administración pública tiene también un protagonismo, pues le compete la difusión de los valores naturales, culturales y paisajísticos del patrimonio público. Por otra parte, la comercialización de productos turísticos es una labor del sector privado.

El indicador de productividad propuesto por los FEDER C009 Aumento del número de visitas previstas a lugares pertenecientes al patrimonio es pertinente para evaluar estas acciones. También el indicador de E035 Número de personas impactadas por las actuaciones de difusión y sensibilización ambiental y de protección y desarrollo del patrimonio natural y cultural (Personas) encajaría bien con las actuaciones propuestas relativas a la promoción (3.3.). De otro lado, tenemos los indicadores identificados para el área de aplicación de Inversión Productiva como: C028 028 Número de empresas subvencionadas para introducir productos nuevos para el mercado (Empresas), E039 Número de personas impactadas por actuaciones de promoción de los sectores económicos en particular los sectores turístico, comercial y culturales (Personas) que podrían ser considerados en este contexto.

\section{CONCLUSIONES}

Como reflexiones finales de este trabajo, se pueden apuntar los siguientes comentarios:

En primer lugar, hay que destacar que la Naturaleza y la Cultura no están lo suficientemente considerados en los Fondos FEDER, teniendo en cuenta que la Unión Europea pre- 
senta un impresionante potencial para el desarrollo basado en estos elementos (solo quedan plasmados en el objetivo temático 6). Si estos temas no aparecen claramente formulados entre los objetivos específicos y las prioridades de inversión será muy complejo que constituyan un motor y catalizador del desarrollo sostenible en los programas nacionales y en sus presupuestos.

Además, en los documentos analizados no se contemplan la Naturaleza y la Cultura desde una misma perspectiva de gestión patrimonial. Si bien desde la visión de los aspectos de conservación y protección se aprecian especificidades que permiten una consideración personalizada, hay que decir, que las estrategias y herramientas de puesta en valor y dinamización socioeconómica pueden abordarse desde una perspectiva conjunta, lo que facilitaría mucho la comprensión y aplicación de los reglamentos y documentos vinculados a los FEDER, donde, en estos momentos, estos aspectos aparecen dispersos en varios objetivos temáticos y diferentes prioridades de inversión. Esta reivindicación no es novedosa, ya que en 2014 se presentó al Consejo de la Unión Europea, la Carta de Roma sobre el capital natural y cultural para reflexionar e introducir las modificaciones necesarias en las políticas de la UE y en los mecanismos de financiación para que se apoyen conjuntamente la gestión del patrimonio natural y cultural en los sitios de la red Natura 2000. La propia Convención de Patrimonio Mundial es un excelente ejemplo de cómo abordar conjuntamente ambos tipos de patrimonio.

Una mención aparte merece el hecho de que el paisaje como tal no aparezca mencionado en estos documentos oficiales, cuando desde otras plataformas comunitarias se intenta impulsar su protección como elemento identitario y transregional del territorio europeo.

Por otra parte, hay que mencionar que si bien se intuye una consideración sistémica, transversal y secuencial de las acciones que se proponen, esto no queda claramente explícito en la redacción de los documentos y sería de gran ayuda clarificar esta situación para que se evidenciara la necesidad de abordar proyectos que van a llevarse a cabo hasta sus últimas consecuencias y no solo van a desarrollar acciones puntuales que no van a poder cumplir ni con los indicadores de productividad establecidos. A este respecto, hay que añadir que la falta de propuestas de acciones relacionadas con la puesta en valor del patrimonio en los FEDER que son fundamentales como fase previa a la dinamización socioeconómica turística y que son garantistas de la buena implantación de actividades turísticas, representa una debilidad estructural muy grave en el conjunto de las prioridades de inversión cuya exclusión puede conducir al desencadenamiento de situaciones de riesgo real para la conservación y protección del patrimonio.

Respecto a los indicadores, es necesario señalar la importancia que tienen para orientar las acciones y actuaciones, por ello tanto los de productividad como los específicos deben plasmar fielmente los resultados. Esto no es una tarea fácil como se ha visto a lo largo del trabajo pero, en especial los de productividad que los fija la Comisión Europea, hay que tenerlos en consideración desde el principio para poder garantizar que se podrán evaluar con ellos las acciones y actuaciones realizadas.

Por otra parte, hay que mencionar la escasa mención que se hace a los procesos participativos, teniendo en cuenta el gran protagonismo que tienen los actores locales en la implantación y desarrollo de proyectos de dinamización turística basados en pequeñas empresas como es el caso que nos ocupa. Los Programas Operativos deberían ser muy claros en este 
sentido. Otro aspecto de gobernanza importante a recordar es la necesaria transversalidad que caracteriza al sector turístico y más cuando además hay que interactuar con el sector medioambiental y el cultural. Esto obliga como se ha mencionado en el trabajo, a una alineación sin fisuras y coordinación clara de todas las administraciones implicadas ya que, de otro modo, es imposible aplicar y llevar a cabo con éxito proyectos europeos.

Por todas estas razones, se estima que la próxima programación europea que se establecerá a partir de 2020, deberá refinar estos contenidos, otorgándole al patrimonio el protagonismo que le corresponde como así se deduce del innumerable conjunto de documentos que avalan la pertinencia de considerarlo como un elemento clave del desarrollo europeo, algunos de los cuales han emanado incluso de la propia Comisión Europea. Si esto no ocurre, como se está observando actualmente con la programación actual, será muy complicado adscribir fondos financieros a acciones y actuaciones que garanticen la conservación y protección del patrimonio europeo y que actúen de motor de la economía.

\section{BIBLIOGRAFÍA}

CANADA'S HISTORIC PLACES (2010): Standards and Guidelines for the Conservation of Historic Places in Canada. Technical report.

CATLING, CH. (2013): Constructive Conservation. Sustainable growth for historic places. Technical report, English Heritage.

CHAPPLE, N. (2012): New uses for former places of Worship. Technical report, English Heritage. COMISIÓN EUROPEA (2010): Europa 2020. Una estrategia para un crecimiento inteligente, sostenible e integrador. Comunicación de la Comisión, Bruselas, 3.3.2010 $\operatorname{COM(2010)} 2020$ final. http://eur-lex.europa.eu/legal-content/ES/TXT/PDF/?uri=CELE X:52010DC2020\&from=ES (consulta: mayo 2016)

COMISIÓN EUROPEA (2014): Towards an integrated approach to cultural heritage for Europe. COM(2014) 477 Communication from the Commission to the European Parliament, the Council, the European Economic and Social Committee and the Committee of the Regions. http://ec.europa.eu/culture/library/publications/2014-heritage-communication_en.pdf (consulta: mayo 2016).

GARCÍA, R., ANDRÉS, J.L. y ESPEJO, C. (2016): “Conocimientos tácitos, transversalidad turística y desarrollo local. El ejemplo de una ciudad desolada y en proceso de reconversión: Lorca (Región de Murcia, España)". PASOS. Revista de Turismo y Patrimonio Cultural, vol. 14, n $^{\circ}$ 5, 1.093-1.107.

HERITAGE OFFICE OF NSW DEPARTMENT OF PLANNING \& THE ROYAL AUSTRALIAN INSTITUTE OF ARCHITECTS NSW (2011): New Uses for Heritage Places. Guidelines for the adaptation of historic buildings and sites. State of New Wales.

ICOMOS (2011): The Paris Declaration on Heritage as a driver of development. Adopted at Paris, UNESCO headquarters, on Thursday 1st December 2011.

LÄHDESMÄKI, T. (2014): “The EU'S Explicit and Implicit Heritage Politics”. European Societies, vol. 16 (3), 401-421.

MINISTERIO DE EDUCACIÓN, CULTURA Y DEPORTE (2012): Plan Estratégico General 2012-2015 de la Secretaría de Estado de Cultura. Secretaría Técnica, Subdirección General de Documentación y Publicaciones. 
MINISTERIO DE EDUCACIÓN, CULTURA Y DEPORTE (2015): Anuario de estadísticas culturales 2015. Secretaría General Técnica. Subdirección General de Documentación y Publicaciones.

MINISTERIO DE HACIENDA Y ADMINISTRACIONES PÚBLICAS DE ESPAÑA (2015): FEDER 2014-2020. Indicadores de Productividad, Programación de programas Operativos. http://www.fomento.gob.es/NR/rdonlyres/4C5F7D8B-4D97-4260-B400E635B12376F8/133955/CatalogoIndicadoresProductividad_20142020.pdf (consulta: enero 2016).

MINISTERIO DE HACIENDA Y ADMINISTRACIONES PÚBLICAS (2014): Acuerdo de Asociación de España 2014-2020. Aprobado por la CE el 30 de octubre de 2014. http://femp.femp.es/files/3580-934-fichero/Acuerdo\%20de\%20Asociaci\%C3\%B3n\%20 de\%20Espa\%C3\%B1a\%202014-2020.pdf (consulta enero 2016).

NACIONES UNIDAS (2015): Transformar nuestro mundo: la Agenda 2030 para el Desarrollo Sostenible. 70 a Asamblea General de Naciones Unidas. http:/www.un.org/ga/ search/view_doc.asp?symbol=A/70/L.1\&Lang=S (consulta: abril 2016)

OECD (2009): The Impact of Culture on Tourism. www.sourceoecd.org/Industryservicestrade/9789264056480 (consulta: abril 2016)

OSIPOVA, E., WILSON, L., BLANEY, R., SHI, Y., FANCOURT, M., STRUBEL, M., SALVATERRA, T., BROWN, C., VERSCHUUREN, B. (2014): The benefits of natural World Heritage: Identifying and assessing ecosystem services and benefits provided by the world's most iconic natural places. Gland, Switzerland: IUCN.

UNITED NATIONS ENVIRONMENT PROGRAMME Y WORLD TOURISM ORGANIZATION (2012): Tourism in the Green Economy. Background Report, UNWTO, Madrid.

VIÑALS, M.J., MORANT, M. y TERUEL, M.D. (2014): “Confort psicológico y experiencia turística. Casos de estudio de espacios naturales protegidos de la Comunidad Valenciana (España)". Boletín Asociación Geógrafos Españoles, nº 65, 293-316.

VIÑALS, M.J., ALONSO-MONASTERIO, P., LOZANO, J.G. Y MARTÍNEZ-SANCHIS, I. (2016): Estudio y elaboración de mapas SIG sobre las inversiones en patrimonio de la Dirección General de Cultura en la provincia de Castellón. Universitat Politècnica de València. Informe inédito. 
